RESEARCH ARTICLE

\title{
Need for closure and compensatory rule-based perception: The role of information consistency
}

\author{
Piotr Dragon (iD \& Małgorzata Kossowska \\ Institute of Psychology, Jagiellonian University, Kraków, Poland
}

\author{
Correspondence \\ Piotr Dragon, Institute of Psychology, \\ Jagiellonian University, Ingardena 6, 31-050 \\ Kraków, Poland. \\ E-mail: dragon.piotr@gmail.com
}

Received: 28 September 2015

Accepted: 22 May 2018

https://doi.org/10.1002/ejsp.2512

Keywords: need for closure, compensatory perception, cognitive inconsistency, group perception

\begin{abstract}
When making comparisons, people tend to use routinized standards, rules, and knowledge structures. Compensatory rules (e.g., "if competent, then cold", "if incompetent, then warm") allow for the quick and easy evaluation of groups when they are compared. We claim that the application of these rules is especially attractive for people who are motivated to seek quick and firm answers (people high in the need for closure-NFC). However, we assume that when people are confronted with expectancy-inconsistent information, higher levels of NFC lead to a lower reliance on these rules. This is because the inconsistency may serve as a signal that the rules no longer provide guidance on how to act. We demonstrated these effects in three studies set in different group contexts, where we manipulated expectancy-consistent and expectancy-inconsistent information. These findings allow for a more comprehensive view of the dynamic and diverse effects of NFC.
\end{abstract}

One important assumption of the lay epistemic theory (Kruglanski, 1989) is that social knowledge is formulated and applied through the use of "if, then" inferential rules, and that social judgments, as any judgments, are made through reasoning from premises to conclusions. The reasoning is syllogistic and includes a major premise ("if $\mathrm{X}$, then $\mathrm{Y}$ ") and a minor premise that provides evidence that the major premise applies to a given case (" $\mathrm{Z}$ is $\mathrm{X}$ " thus " $\mathrm{Z}$ is $\mathrm{Y}$ ") (Kruglanski, Dechesne, Orehek, \& Pierro, 2009; Kruglanski, Pierro, Mannetti, Erb, \& Chun, 2007; Kruglanski \& Shteynberg, 2012). For example, such reasoning can be seen in the belief that "if mother, then warm" (major premise), paired with the belief that because a given woman has children (minor premise), she must be warm (conclusion). Such knowledge is routinized through practice, and becomes easily accessible (Kruglanski \& Shteynberg, 2012; Neal, Wood, \& Quinn, 2006).

Research has shown that when making comparisons people tend to use such routinized standards, rules, and knowledge structures (Mussweiler \& Rüter, 2003; Rüter \& Mussweiler, 2005) which allows them to save cognitive resources, to reduce uncertainty (Mussweiler \& Posten, 2011), and at the same time to reach a solution quickly and accurately (Mussweiler \& Epstude, 2009). An example of such rule-based social perception when making comparisons can be seen in evaluations of two groups on the dimensions of warmth and competence (e.g., Kervyn, Yzerbyt, Demoulin, \& Judd, 2008). In that situation, when people perceive one group as being higher on one dimension (e.g., competence), they tend to compensate and perceive the other group as being higher on the other dimension (e.g., warmth). This effect can be explained as an application of simple rules ("if competent, then cold" or "if incompetent, then warm"). Such rules can be used with minimum cognitive resources and may be easily applied in many different contexts and situations; thus they may help to efficiently organize social perception.

A considerable amount of research has shown that the need for closure (NFC), defined as a feeling of discomfort experienced in the face of cognitive uncertainty (Kruglanski, 1989), motivates people to use routinized standards, rules, and knowledge structures (e.g., Kruglanski et al., 2009). Although previous studies on NFC have mainly focused on the reliance on pre-existing knowledge structures in social perception (e.g., stereotypes, beliefs, social schemas), to date there has been no study examining rule application. This article is aimed at filling this gap. We focus on compensatory rules, as these rules are seen as cognitive tools that may efficiently simplify cognition. This is because compensatory rules (e.g., "if competent, then cold", "if incompetent, then warm") allow for the quick and easy evaluation of groups when they are compared. Information about a group on one dimension allows for inferences to be made about the group on the other important dimension (e.g., information that the group is competent means that the group is also cold). We claim that the application of such rules is especially 
attractive for people who are motivated to seek quick and firm answers (such as individuals high in NFC) and that they are less attractive for people who prefer to process information more exhaustively (individuals low in NFC). Therefore, when forming impressions of groups in comparative contexts, individuals high in NFC should apply compensatory rules to a greater extent than would those low in NFC. However, the question arises whether this is always the case.

\section{Expectancy-Inconsistent Information, the Application of Compensatory Rules, and the Need for Closure}

The application of previously learned standards, rules, and knowledge can serve as a heuristic for evaluating social objects, which is usually attractive for people who are high (vs. low) in NFC (Driscoll, Hamilton, \& Sorrentino, 1991; Kruglanski et al., 2009). However, research has shown that individuals who are high in NFC sometimes do not rely on pre-existing knowledge structures, but rather engage in complex, bottom-up cognition (for an overview, see Roets, Kruglanski, Kossowska, Pierro, \& Hong, 2015). For instance, higher levels of NFC induced a heightened level of information processing when initial confidence was low, as opposed to when initial confidence was high (Kruglanski, Peri, \& Zakai, 1991). Also, Van Hiel and Mervielde (2002) showed that NFC intensified information acquisition when ambiguous information was included in the judgmental process. Likewise, Klein and Webster (2000) demonstrated that people high in NFC processed a message systematically when heuristic cues were not available. In a similar vein, Houghton and Grewal (2000) showed that high levels of NFC led to a less extensive information search in a consumer choice paradigm, but only when participants had a previously formed attitude about the product.

These studies demonstrate that NFC is associated with applying previous knowledge, but only when there is an initial, satisfactory basis for closure, resulting from familiarity with a subject or from having sufficiently strong confidence in one's initial opinion. If people who are high in NFC lack a knowledge base on which they can rely with relative confidence (e.g., when their self-view is threatened by experiences of powerlessness, uncontrollability, or self-image threat) they can desist from their default, heuristic, and simplistic way of information processing and use a more effortful and systematic one (e.g., Kossowska, Bukowski, Guinote, Dragon, \& Kruglanski, 2016; Kossowska, Guinote, \& Strojny, 2016 for a review see, Roets et al., 2015). This means that individuals high in NFC do not necessarily always have to be cognitive misers, rigidly relying on what they already know. The main motivation of people high in NFC is to reach closure, thus to reduce uncertainty (Kruglanski, 1989). People high in NFC usually rely on pre-existing knowledge structures that were tried and proven to be useful in the past, as these means are associated with greater expectancy of successful closure. However, in situations when their trust in pre-existing knowledge structures is undermined and the expectancy of fulfilling the goal decreases, people high in NFC should be more willing to search for alternative means for achieving closure, and they should therefore consider new strategies for doing so, even if they are more effortful. This is consistent with the original formulation of NFC theory (Kruglanski, 1989), where high levels of the NFC are associated with the motivational readiness to invest effort, rather than withdraw effort, in order to attain the goal of certainty. In other words, if necessary, people high in NFC can rise to the challenge posed by the environment, by adapting to the unusual situation and by using more effortful means in order to achieve their focal goal, that is to reduce uncertainty.

In this article, we examine the link between NFC and the application of compensatory rules, a link that has yet to be tested. We assume that when two groups are being compared, people high in NFC, when provided with information consistent with their previous knowledge about groups, will efficiently apply compensatory rules. However, if exposed to information that is distinct from, inconsistent with, and even contradictory to their knowledge, they may not apply routinized rules when forming impressions about groups. Instead, they may attend to and examine more information. As a consequence of this, such people may develop a more sophisticated perception of the groups, instead of relying on simple compensatory perception. This is because exposure to information that is strongly inconsistent with important knowledge could reduce the expectancy of attaining closure via typical means, that is, heuristics, schemas, stereotypes, or-as in the case of the research described in this article-compensatory rules. Thus, we assume that when provided with stereotypeinconsistent information, individuals high in NFC will apply routinized heuristic tools to establish relationships between social objects (i.e., compensatory rules) to a lesser extent, as compared to when there is no inconsistency.

Above we referred only to people high in NFC. This is because we do not expect that people who are low in NFC will be affected by the inconsistency manipulation. This is because, by definition, people low in NFC do not strive for predictability or order. On the contrary, they embrace ambiguity instead of certainty. Also, as opposed to people who are high in NFC, people who are low in NFC, as a default, process information exhaustively, systematically, and effortfully, and they rely on pre-existing knowledge structures to a lesser extent than people who are high in NFC (Kruglanski, 1989). Thus, we do not expect that when experiencing inconsistency, people who are low in NFC will change their default style of information processing, as inconsistency does not pose a threat to them, as it does in the case of people high in NFC. 


\section{Overview of the Study}

To test our hypotheses, we conducted three studies. In Studies 1 and 2, we used a procedure similar to that described by Kervyn et al. (2008) in which participants evaluated two nationalities on the dimensions of warmth and competence. The target nationality was the nationality of the participants, while the comparison nationalities were either low in competence/high in warmth (Study 1), or high in competence/low in warmth (Study 2). The comparison groups were chosen on the basis of previous research (CBOS, 2013; Dolińska \& Fałkowski, 2011) and their dimensional fit was additionally tested in a pilot study (see Supporting Information). Moreover, we provided participants with either expectancy-consistent (Study 1) or expectancy-inconsistent (Study 2) information. In both studies, the manipulated information referred to a national group's position on the dimension of warmth. In Study 3 we tested all conditions in a single study by providing expectancyconsistent information, expectancy-inconsistent information, or no information at all (control condition). In this study, we used a different group context: Instead of national groups we used occupational groups. Furthermore, instead of manipulating consistency/inconsistency on the warmth dimension as in Studies 1 and 2, we manipulated the dimension of competence. This allowed us to test the generalizability of the predicted effects in a different context.

\section{Study 1}

The aim of Study 1 was to test whether NFC predicts the application of compensatory rules when forming impressions about two groups. Participants evaluated two nationalities on the dimensions of warmth and competence. The target nationality was the nationality of the participants: Polish. On the basis of previous research (e.g., Dolińska \& Fałkowski, 2011) and a pilot study (see Supporting Information), Spaniards were chosen as the comparison nationality that is stereotypically perceived in Poland to be warm but not competent. We assumed that when participants high (vs. low) in NFC obtained expectancy-consistent information (that a group stereotypically perceived as warm, is in fact warm), as well as when they were in the control condition in which they do not obtain any expectancy-consistent nor expectancy-inconsistent information, they would rely on their routinized compensatory rules. As a consequence, the more participants who are high in NFC perceive Spaniards as being warmer than Poles, the more they should perceive Poles as being more competent than Spaniards.

\section{Design and Participants}

This study was a 2 (consistency manipulation: consistency vs. control) between-subjects design. All participants evaluated Spaniards and Poles on the dimensions of warmth, competence, and morality. The order of the presentation of a given nationality was counterbalanced.

The experiment was conducted online. Eighty users ${ }^{1}$ of an online research panel took part in the study, but nine of them failed to prove that they had familiarized themselves with the experimental manipulation and were thus excluded from further analyses. Thus, we used data from 71 participants (32 in experimental condition, 39 in the control condition; 52 women, 19 men; $\left.M_{\text {age }}=27.31, S D=8.27\right)$. They were compensated three PLN (approximately one USD) for completing the questionnaire.

\section{Materials and Procedure}

Participants completed a short version of the Need for Closure Scale (Webster \& Kruglanski, 1994). We used the following subscales in our analysis: preference for order and structure in the environment, predictability of future contexts, and affective discomfort occasioned by ambiguity. We removed the subscales closed-mindedness and decisiveness from analysis, as they were not correlated with any other subscale. ${ }^{2}$ This is in line with previous research establishing that the decisiveness subscale measures ability rather than motivation (Roets \& Van Hiel, 2007), and that the closed-mindedness subscale captures a different phenomenon (Neuberg $\&$ Newsom, 1993). The reliability of the scale was $\alpha=$.83. Items were averaged into an index of NFC $(M=4.11 ; S D=0.70)$. The higher the index, the higher the score on NFC.

Participants were then randomly assigned to one of two conditions: the experimental condition or the control condition. In both conditions, participants were asked to carefully read the text presented to them. In the experimental condition, participants read the following text:

'The Eurobarometer is a representative survey of public opinion, organized on behalf of the European Commission. 40,000 citizens from all member countries of the European Union participated in the last

\footnotetext{
${ }^{1}$ We did not perform an a-priori power analysis before conducting our studies. It was because studies were conducted four years ago, when it was not yet a common research practice. Nevertheless, we believe that despite this limitation - that we fully acknowledge-the presented results are reliable. Obtained results are consistent across three independent studies. Moreover, as a post-hoc power analysis is generally not recommended (e.g., Hoenig \& Heisey, 2001; Zumbo \& Hubley, 1998), in order to at least partially control for potential problems that conducted studies were underpowered, we performed a meta-analysis (e.g., Cohn \& Becker, 2003; Higgins \& Green, 2011).

${ }^{2}$ Across all three studies reported in this article, closed-mindedness and decisiveness subscales did not correlate with the other subscales (preference for order and structure in the environment, predictability of future contexts, and affective discomfort occasioned by ambiguity); therefore in all experiments we used the NFC score based on the three subscales.
} 
survey about the "self-portraits" of Europeans. This research investigated, among other issues, what the citizens of European Union member countries think about each other. In one of the questions, the respondents were asked to choose "the most likeable nationality in the European Union." Respondents could not vote for their own country (e.g., people from Poland could not vote for Poles, etc.). The results of the study clearly ascertained that among Europeans the most likeable nation in the European Union are the Spanish $164 \%$ of respondents voted for people from Spain, from among the 27 possible countries). The research in which you are currently participating is an additional study of the findings from the Eurobarometer, focusing on two nationalities'.

In the control condition the text was exactly the same; however, the underlined portion was removed. Subsequently, participants evaluated people from Spain and Poland on the dimensions of warmth, competence, and morality. As researchers have argued that morality is also an important dimension in impression formation (e.g., Abele, Uchronski, Suitner, \& Wojciszke, 2008; Abele \& Wojciszke, 2007; Wojciszke \& Abele, 2008), we controlled for its influence. The items used in this study were: warm, friendly, helpful (for warmth), competent, capable, intelligent (for competence), and moral, honest, good (for morality). Participants assessed these traits on a seven-point scale ( $1=$ not at all; $7=$ very much). Being concerned about the possible effects of social desirability and aiming to capture broader societal perceptions, following Fiske, Cuddy, Glick, and Xu (2002), indirect instructions were used. Participants were asked how the groups are perceived by Polish society ("To what extent, in your opinion, do people in Poland perceive people from [Spain, Poland] as..."). Reliabilities for the scales were: Spain warmth $\alpha=.88$; Spain competence $\alpha=.83$; Spain morality $\alpha=.84$; Poland warmth $\alpha=.85$; Poland competence $\alpha=.86$; Poland morality $\alpha=.85$. The items related to a given dimension were averaged into a single index. The higher the index, the higher the group was rated on a given dimension.

Afterwards, participants from the experimental condition answered two questions on seven-point scales ( $1=$ not at all; $7=$ very much $)$ that assessed the effectiveness of the manipulation (i.e., its consistency with previous stereotypical knowledge). They were asked to what extent the Eurobarometer findings about Spaniards were surprising and to what extent they were expected. Participants then assessed their identification with Poland on a seven-point scale, using a pictorial measure similar to the method described by Swann, Gómez, Seyle, Morales, and Huici (2009). Because one of the evaluated groups was the national group of the participants, their identification with the country might affect the evaluations. Thus, we controlled for the strength of identification in order to rule out its influence $(M=4.21 ; S D=1.72)$.
Finally, all participants answered two questions that assessed whether they had read the cover story. Because the study was conducted online, there was limited control over the participants. Therefore, it was important to incorporate an additional index that would allow us to distinguish between reliable and unreliable participants. All participants were asked how many people took part in the Eurobarometer survey, and additionally, participants from the experimental condition were asked which nationality was reported to be the most likable in the survey. Nine participants failed to answer these questions (eight from the experimental condition, and one from the control condition), and were excluded from further analyses. All of these participants also spent a significantly lower amount of time completing the survey, as compared to the average (nine minutes). Thus, we used data from 71 participants. After completing the questionnaire, participants were debriefed.

In order to observe whether the compensatory rules were applied, we calculated the indexes of the differentiation between groups ${ }^{3}$ (Kervyn et al., 2009). The index of the differentiation between the groups on the warmth dimension was calculated as the mean evaluation of Poles subtracted from the mean evaluation of Spaniards. The index of the differentiation between the groups on the dimension of competence was calculated as the mean evaluation of Spaniards subtracted from the mean evaluation of Poles on this dimension. Thus, for the dimension of warmth, positive values of the differentiation index indicate that Spaniards were evaluated as being warmer than Poles, while for the competence dimension, positive values mean that Poles were evaluated as being more competent than Spaniards. A positive relation between these two indexes would indicate that the compensatory rules had been applied (the more one group was perceived as being higher on one dimension, the more the second group was perceived as being higher on the other dimension). The mean values for these indexes were: for differentiation on the dimension of warmth $(M=1.31 ; S D=1.27)$, and for differentiation on the competence dimension $(M=0.48 ; S D=1.51)$.

\footnotetext{
${ }^{3}$ We used difference scores, as it is a way to evaluate compensatory perception at an individual level, when comparison context is involved (two groups are evaluated). As has been shown in previous research on compensatory perception, in comparative context the mutual position of two groups on one dimension (e.g., one group being perceived as more competent than the other) affects perception of these groups on a second dimension (the group being perceived as less competent is also perceived as being higher on warmth dimension) (e.g., Kervyn, Judd, \& Yzerbyt, 2009). Therefore, it has to be taken into consideration when analyzing results, and the way to achieve it is to analyze difference scores. In this way, it is possible to evaluate the degree to which participants differentiate between groups on a given dimension (whether they in fact perceive a given group as being higher on a given dimension as compared to the other group, e.g., Spaniards as being more warm than Poles) and how it is related to the mutual position of these groups on the other dimension.
} 
We predicted that in both conditions, that is, when provided with information that is consistent with previous knowledge (consistency condition) or not provided with any information about the group (control condition), people high in NFC would apply the compensatory rules to a greater extent than people low in NFC. We did not expect any differences between the experimental and control conditions, because in both situations, the information given does not violate preexisting knowledge, thus participants should act based on their preferred processing mode. We expected an interaction between the predictor measure (the differentiation between Spaniards and Poles on the dimension of warmth) and NFC, on the dependent variable (the differentiation between Poles and Spaniards on the competence dimension). In addition, we controlled for the evaluations on the morality dimension and the strength of participants' identification with their Polish nationality.

\section{Results and Discussion}

Manipulation check. As predicted, the information about the high ratings of the Spanish nation on the dimension of warmth was statistically significantly more expected $(M=5.03 ; S D=1.60)$ than surprising $(M=2.69 ; S D=1.58), t(31)=4.48, p<.001, d=$ 1.47. ${ }^{4}$ This means that participants perceived the information provided in the manipulation as being consistent with their previous knowledge; thus the manipulation induced the experience of consistency.

Need for closure and the application of compensatory rules. In order to test our hypotheses, a regression analysis was performed. We tested the full model with all of the variables listed above included in the model: evaluations of Poles and Spaniards on the morality dimension, strength of participants' identification with their Polish nationality, all the main effects, the two-way interaction combinations, and a threeway interaction between predictor (the differentiation between Spaniards and Poles on the dimension of warmth) and two moderators (i.e., NFC and the dummy coded conditions: 0 control/1 consistency condition). The DV was the differentiation between Poles and Spaniards on the competence dimension. All continuous variables were standardized before the analysis. In this and all analyses in this and the following studies, reported coefficients and confidence intervals refer to standardized coefficients. The whole model was significant, $F(10,59)=3.16, p=.003, R^{2}=.35$. The moral evaluation of Poles $(\beta=.43 ; t=3.51$, $p=.001,95 \%$ CI $[0.19,0.68])$ and the moral evaluation of Spaniards $(\beta=-.32 ; t=2.74, p=.008,95 \%$ CI $[-0.55,-0.09])$ turned out to be significant. Also, the

\footnotetext{
${ }^{4}$ Manipulation checks in all conducted studies were performed only in experimental conditions, and degrees of freedom correspond to number of participants in experimental conditions.
}

main effects of the warmth differentiation index $(\beta=.36 ; t=1.78, p=.082,95 \%$ CI $[-0.05,0.77])$ and the strength of identification $(\beta=.23 ; t=1.90$, $p=.062,95 \%$ CI $[-0.01,0.47])$ were marginally nonsignificant. As predicted, the three-way interaction was not statistically significant $(\beta=-.41 ; t=1.64$, $p=.106,95 \%$ CI $[-0.91,0.09])$, and the interaction term between NFC and the warmth differentiation index was significant $(\beta=.49 ; t=2.37, p=.021$, $R^{2}=.06,95 \%$ CI $\left.[0.08,0.90]\right)$. The interaction is depicted in Figure 1. To probe for a significant interaction, we used a simple slope analysis and calculated the effect of our predictor on our DV at low $(-1 S D)$ and high $(+1 S D)$ values of the moderator (NFC). A simple slope analysis indicated a positive relation between the warmth differentiation index (predictor), and the competence differentiation index (DV) among high NFC participants $(\beta=.85 ; t=2.40, p=.020,95 \%$ CI $[0.14$, 1.56]), but not among low NFC participants $(\beta=-.13$; $t=.61, p=.544,95 \%$ CI $[-0.54,0.29])$.

In sum, the more people high in NFC differentiated between people from Spain and Poland on the dimension of warmth (i.e., the higher Spaniards were evaluated on the dimension of warmth as compared to Poles), the more they differentiated between them on the dimension of competence in the opposite direction (i.e., the higher Poles were evaluated on dimension of competence as compared to Spaniards). Thus, in line with expectations, this reveals that people high (vs. low) in NFC applied the compensatory rules when evaluating two groups in a comparative context. This effect occurred when no information about Spaniards was provided, but also when participants were provided with expectancy-consistent information. This shows that when there is no inconsistency with one's previous beliefs, people high in NFC apply routinized rules to resolve the task, while people low in NFC do not rely on these rules as heavily.

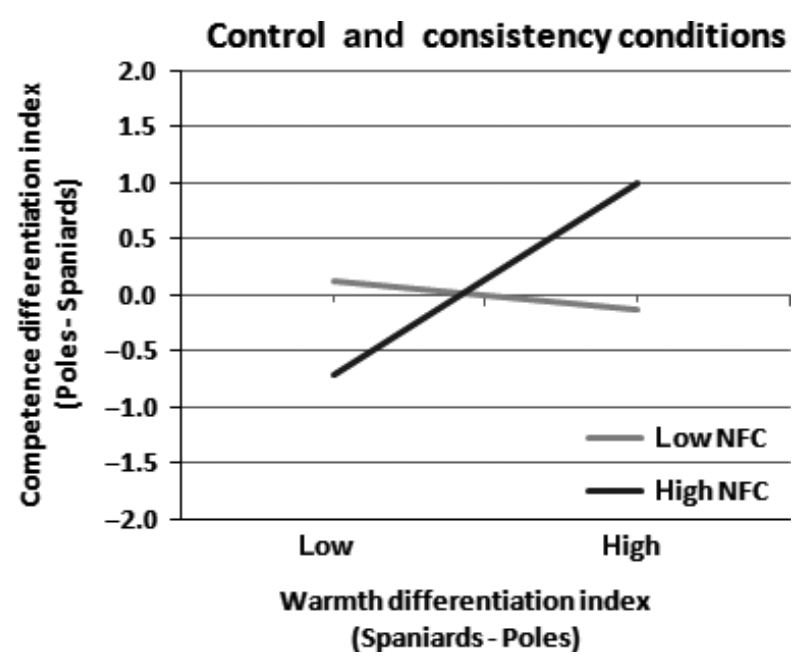

Fig. 1: Regression lines demonstrate application of the compensatory rule (differentiation between Poles and Spanish people on the dimension of competence, as a function of differentiation between these groups on dimension of warmth) and need for closure (NFC) 


\section{Study 2}

The aim of Study 2 was to test whether, relative to the control condition, expectancy-inconsistent information provided to participants leads to lower reliance on compensatory rules among people high (vs. low) in NFC. As in Study 1, participants evaluated two nationalities on the dimensions of warmth and competence. The target nationality was the nationality of the participants (Poles). On the basis of previous research (e.g., Dolińska \& Fałkowski, 2011), Germans were chosen as the comparison nationality perceived in Poland as being competent and cold. In Study 2, instead of providing information consistent with previous knowledge, inconsistent information was provided. We assumed that in the control condition (when no information regarding Germans is provided), participants high in NFC would apply the compensatory rules to a greater extent than participants low in NFC (as in Study 1). As a consequence, the more participants high in NFC perceive Germans as being more competent than Poles, the more they should perceive Poles as being warmer than Germans. However, in the experimental condition the experience of inconsistency should discourage participants high in NFC from using their routinized rules; thus this effect should disappear.

\section{Design and Participants}

This study was a 2 (consistency manipulation: inconsistency vs. control) between-subjects design. All participants evaluated people from Germany and Poland on the dimensions of warmth, competence, and morality. The order of the presentation of a given nationality was counterbalanced.

The study was conducted online, using an online research panel. Sixty-six participants took part in the study. Ten participants failed to prove that they had familiarized themselves with the experimental manipulation and were therefore excluded from further analyses. Thus, we used data from 56 participants (28 in the experimental condition, 28 in the control condition; 38 women, 18 men; $M_{\text {age }}=28.07, S D=8.97$ ). Participants received three PLN (approximately one USD) for completing the questionnaire.

\section{Materials and Procedure}

All the materials were exactly the same as in Study 1 (except the experimental manipulation), and were presented in the same order. Firstly, participants completed a short version of the NFC Scale. As before, three subscales were used in the analyses (Cronbach's $\alpha=.81)$. Items were averaged into an index of NFC; the higher the score, the higher the levels of NFC $(M=4.18 ; S D=0.62)$. Secondly, participants were randomly assigned to either the experimental or the control condition. The manipulation was identical to that in Study 1, only in the experimental condition people read that the most likeable nation in the European Union are the Germans.

Subsequently, participants evaluated Germans and Poles on warmth, competence, and morality, using the same items as in Study 1. Reliabilities for the scales were as follows: Germans' warmth $\alpha=.86$; Germans' competence $\alpha=.85$; Germans' morality $\alpha=.81$; Poles' warmth $\alpha=.79$; Poles' competence $\alpha=.86$; Poles' morality $\alpha=.86$. Items related to a given dimension were averaged into a single index. As in Study 1, participants in the experimental condition then answered two questions assessing the effectiveness of the manipulation, that is, its inconsistency with previous (stereotypical) knowledge. We asked to what extent the results of the Eurobarometer were surprising and to what extent they were expected.

Participants then assessed their identification with their country (Poland) $(M=4.16 ; S D=1.54)$ using the same method as in Study 1, and they answered two questions assessing whether they had read the manipulation. Ten participants failed to answer these questions correctly (four from the experimental condition, and six from the control condition), and were excluded from further analyses. Thus, we used data from 56 participants. After completing the questionnaire, participants were debriefed.

As in Study 1, the application of compensatory rules was assessed by using indices of the differentiation between groups on a given dimension. For the warmth dimension we subtracted the mean evaluation of Germans from the mean evaluation of Poles, and for the competence dimension we subtracted the mean evaluation of Poles from the mean evaluation of Germans on this dimension. As Germans are stereotypically perceived as being competent and cold, in order to obtain indices comparable to those in Study 1 (i.e., a positive value of the index indicating that one group is stereotypically perceived as being higher on the dimension than the second group), the sequence of subtraction was reversed. Thus, for the competence dimension, a positive value indicates that Germans were evaluated as being higher on competence than Poles, while for the warmth dimension, a positive value of the differentiation index indicates that Poles were evaluated as being higher on warmth than Germans. A positive relation between these two indexes indicates the application of the compensatory rules. The mean values for these indices were as follows: for the differentiation on the dimension of warmth $(M=1.20 ; S D=1.32)$, and for the differentiation on the dimension of competence $(M=0.58 ; S D=1.41)$.

We expected that in the control condition, people high (but not low) in NFC should apply the compensatory rules, while in the experimental condition (inconsistency condition) this effect should disappear among participants high in NFC. Thus, in this study we expected a three-way interaction (predictor $\times$ NFC $\times$ experimental condition) to be statistically significant. The dependent variable was the differentiation on the warmth dimension, and the 
predictor variable was the differentiation on the competence dimension (the dimension on which Germans are perceived as being higher than Poles). Also, as before, we controlled for moral evaluations and the strength of national identification (with Poland).

\section{Results and Discussion}

Manipulation check. As predicted, the information about the high position of Germans on the dimension of warmth was significantly more surprising $(M=5.07 ; S D=1.36)$ than expected $(M=3.07$; $S D=1.36), t(27)=4.05, p<.001, d=1.47$. This indicates that participants perceived the information provided as inconsistent with their previous knowledge; thus the manipulation was successful and induced the experience of inconsistency.

Need for closure and the application of compensatory rules. As in Study 1, a regression analysis was performed. Once again, we tested the full model with all the variables listed above included in the model: evaluation of Poles and Germans on the dimension of morality, the strength of national identification, all the main effects, the two-way interaction combinations, and a three-way interaction between predictor (the differentiation between Germans and Poles on the dimension of competence) and two moderators (i.e., NFC and dummy coded experimental condition: 0 control/1 inconsistency condition). The DV was the differentiation between Poles and Germans on the warmth dimension. All variables were standardized before the analysis. The model was significant, $F(10,45)=4.59, p<.001, R^{2}=.51$. Morality evaluations of both Poles $(\beta=.46 ; t=3.28, p=.001$, $95 \%$ CI $[0.18,0.75])$ and Germans $(\beta=-.43$; $t=3.75, p=.002,95 \%$ CI $[-0.67,-0.20])$ were statistically significant, as was the interaction between the index of competence differentiation and NFC $(\beta=.46 ; t=2.29, p=.027,95 \%$ CI $[0.06,0.89])$. As predicted, a three-way interaction between NFC, condition, and the competence differentiation index (predictor) was statistically significant $(\beta=-.74 ; t=3.09$, $p=.003, R^{2}=.11,95 \%$ CI $\left.[-1.23,-0.26]\right)$. The interaction is depicted in Figure 2.

To probe for a significant interaction, we used a simple slope analysis and calculated the effect of our predictor on our DV at low $(-1 S D)$ and high (+1 $S D)$ values of the first moderator (NFC), at values of a second moderator (dummy coded experimental condition). A simple slope analysis indicated that in the control condition, a positive relation between the competence differentiation index (predictor) and the warmth differentiation index (DV) appeared among participants high in NFC $(\beta=.85 ; t=2.42, p=.020$, $95 \%$ CI $[0.14,1.55])$, but not among participants low in NFC $(\beta=-.10 ; t=.39, p=.696,95 \%$ CI $[-0.64$, $0.43])$. In the experimental condition, we found a positive, although non-significant, relation between the competence differentiation index and the warmth differentiation index among participants low in NFC $(\beta=.21 ; t=1.08, p=.285,95 \%$ CI $[-0.18,0.60])$, but it was negative and non-significant among participants high in NFC $(\beta=-.32 ; t=1.87, p=.069,95 \%$ CI $[-0.67,0.03])$.

In sum, in the control condition, the more people high in NFC differentiated between Germans and Poles on the dimension of competence (the higher Germans were evaluated on the dimension of competence relative to Poles), the more they differentiated between them on the dimension of warmth in the opposite direction (the higher Poles were evaluated on the dimension of warmth relative to Germans). Thus, in line with expectations, this reveals that people high (vs. low) in NFC apply the compensatory rules when evaluating two groups in a comparative context, but this effect occurred only in the condition where no information about Germans was provided (control condition). When expectancy-inconsistent information was provided, people both high and low in NFC did not apply previously learned rules to resolve the task. This is in line with our assumption that, when confronted with inconsistent information, people high in NFC become less confident that their knowledge is accurate and reliable and as a result they deviate from relying on accessible knowledge structures. Thus, they
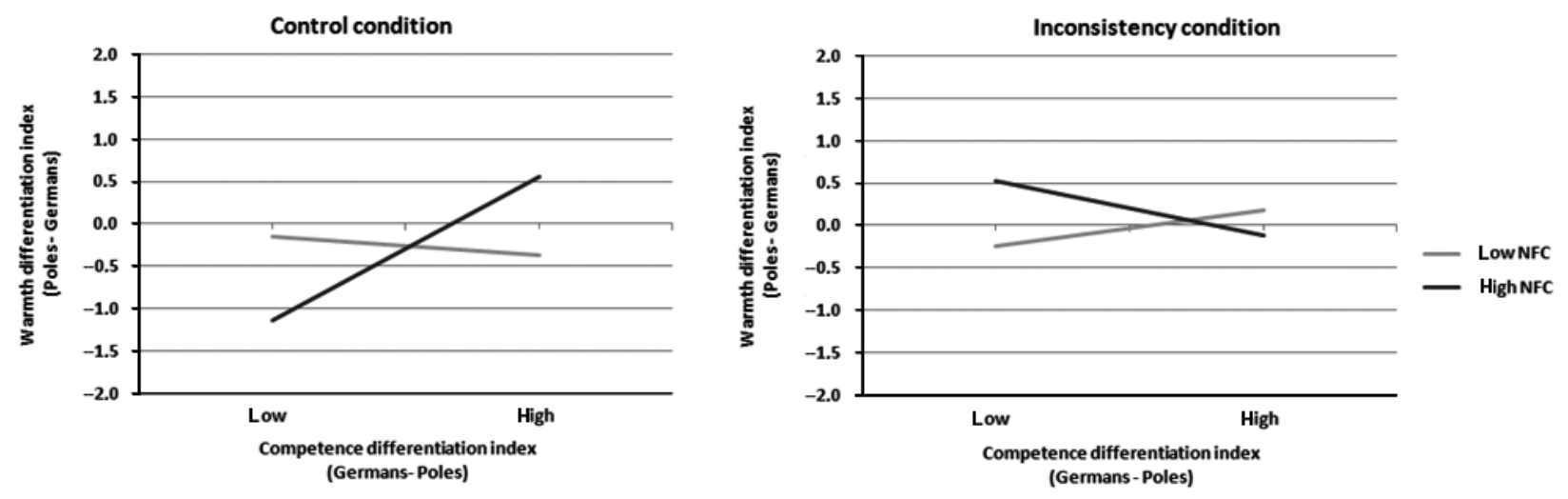

Fig. 2: Regression lines demonstrate application of the compensatory rule (the differentiation between Poles and Germans on the dimension of warmth, as a function of differentiation between these groups on dimension of competence), need for closure (NFC), and experimental condition 
become more sensitive to non-stereotypical information that in consequence serves them as a basis for intergroup evaluations.

In Studies 1 and 2, the comparison groups were national groups, and the manipulation referred to the dimension of warmth. Moreover, participants belonged to one of the two evaluated groups. Thus, while we controlled for the strength of identification with the ingroup, it could be argued that some sort of ingroup processes might be affecting the evaluations. Thus, in order to show that the effect is not specific to the context used in Studies 1 and 2, in Study 3 we changed the group context, as well as the manipulated dimension.

\section{Study 3}

In Study 3, we provided control, expectancy-inconsistency, and expectancy-consistency conditions. We manipulated the dimension of competence, instead of warmth as in the previous experiments. Also, instead of national groups (including the participants' ingroup), occupational groups were used. This allows us to test the generalizability of the predicted effects in a different social context. We also tested whether changes in the perceptions of a given group on one dimension (e.g., as the result of an anti-stereotype intervention) could change the evaluation of the second group in the opposite direction on the second dimension. In that way, we investigated not only whether the compensatory rules were applied but also whether inconsistency may change intergroup perception.

Thus, this was a repeated measure experiment, ${ }^{5}$ in which we measured the evaluations of two groups (pretest), then participants obtained information

\footnotetext{
${ }^{5}$ Moreover, because it was not certain whether the perception of a group can be changed simply by obtaining information inconsistent with previous knowledge, we included an additional manipulation. In two conditions, participants obtained information consistent/inconsistent with stereotypical knowledge (that a given group scored very high on an intelligence test), and in two conditions in addition participants were informed that their own group scored poorly on this test. We assumed, that obtaining information that not only the group perceived as being stereotypically incompetent had scored high on intelligence test, but also that the participants' own group had scored poorly may additionally strengthen the manipulated experience of inconsistency. In every analysis described in this study we controlled for the influence of this additional manipulation, although it turned out there was no significant effect associated with this manipulation; therefore for the clarity of reasoning and presentation we do not describe it later on. But because of this additional manipulation, participants answered also to an Importance to Identity subscale from the Collective Self-Esteem Scale (Luhtanen \& Crocker, 1992). This subscale was used to control for the strength of participants' identification with their school class, which can affect the evaluations of the participants. This subscale consisted of four items (e.g., "The school class I belong to is an important reflection of who I am."), on which participants answered using a seven-point scale $(1=$ completely disagree $7=$ completely agree). The scale was reliable, $\alpha=.79$. Items were summed into an index $(M=15.51 ; S D=4.32)$. In the analyses we controlled for this variable but it did not reveal any significant results.
}

consistent or inconsistent with stereotypical knowledge (experimental conditions) or no information (control condition), and again they evaluated these groups (posttest).

\section{Design and Participants}

This study has a mixed between-within subjects design, with one within-subjects variable: the evaluation moment (before the manipulation vs. after the manipulation), and one between-subjects variable: the consistency manipulation (consistency vs. inconsistency vs. control). All participants evaluated two groups (business school students and culinary school students) on the dimensions of warmth, competence, and morality, before and after the manipulation. The order of the presentation of a given group was counterbalanced.

In total, 105 high school students (34 in the consistency condition, 37 in the inconsistency condition, 34 in the control condition; 65 women, 40 men; $M_{\text {age }}=17.37 ; S D=0.55$ ), from six classes participated in the study. Classes were randomly assigned to one of three between-subjects conditions.

\section{Materials and Procedure}

An experimenter explained to the students that the study consists of two parts. In the first part, participants evaluated two groups on warmth, competence and morality, using the same items as in the previous studies. The first target group was a group perceived as being warm and incompetent (culinary school students), while the second group was a group perceived as being competent and cold (business school students). As in the previous studies, items related to a given dimension were averaged into a single index. The reliabilities for the resulting scales were as follows: culinary school warmth $\alpha=.89$; culinary school competence $\alpha=.86$; culinary school morality $\alpha=.82$; business school warmth $\alpha=.86$; business school competence $\alpha=.81$; business school morality $\alpha=.84$.

As in Studies 1 and 2, participants completed a short version of the NFC Scale. The same three subscales as in the previous experiments were used in further analyses. The items were averaged into a single index of NFC $(M=3.93 ; S D=0.70)$, which formed a reliable scale, $\alpha=.74$.

Finally, in order to create a reliable comparison context, participants completed a bogus intelligence test. To maximize the perceived relevance of the test, the experimenter emphasized that the test was well validated on a sample of more than 100,000 individuals, that this version was designed especially for high school students, and that the results were highly predictive of high school completion, future academic performance, and employment prospects. The test was comprised of seven tasks (similar to Raven's Matrices), and participants had 7 minutes to solve them. After 
completing the test, participants were informed that they would be receiving the results a week later when taking part in the next step of the study.

In the second part of the study, which took place a week later, we introduced the manipulation. At the start of this part an experimenter informed the participants that he did not have enough time to prepare individual feedback on the previous intelligence test. In all conditions, except the control condition, participants were told that although they would not be receiving their individual results, they would receive information about the average results from various high schools where the same test was recently administered. They were also informed that the test was highly valid and reliable. In the inconsistency condition, participants were informed that culinary high school students (a group stereotypically perceived as being warm but incompetent) scored very high on the test. In the consistency condition, participants were informed that business school students (a group stereotypically perceived as being cold but competent) had scored very high on the test.

Subsequently, participants once again evaluated business school students and culinary school students on the same items as in the first part of the study. Then, participants from the experimental conditions (consistency and inconsistency) answered two questions (on a seven-point scale) assessing the effectiveness of the manipulation; namely, the extent to which the results of culinary school and business school students were surprising and to what extent they were expected. Finally, participants were debriefed. The experimenter made sure that the participants understood that the received feedback regarding the test results was bogus and that the test itself did not measure intelligence.

We assessed change in the perception of culinary school students on the dimension of warmth (predictor), and change in the perception of business school students on the dimension of competence (dependent variable). Thus, the compensatory rules application was indicated by a positive relationship between the change in the perception of one group on one dimension (culinary school students being perceived as warmer after the manipulation, as compared to before the manipulation), and the change in the perception of the other group on the other dimension (business school students being perceived as more competent after the manipulation, as compared to before the manipulation). We assumed that in the control condition (when no information regarding the evaluated groups was provided), and in the consistency condition, the compensatory rules would be applied by participants high but not low in NFC (similar to Study 1). We also assumed that this effect would disappear among high NFC participants when expectancy-inconsistent information was provided (similar to Study 2).

\section{Results and Discussion}

Manipulation check. In order to test whether the information regarding the high score of culinary school students on the dimension of competence was surprising for participants, and thus inconsistent with their expectations, an ANOVA was conducted with one within-subjects variable: expectation (expected vs. surprising), and one between-subjects variable: consistency manipulation (consistency vs. inconsistency). Only the interaction term between the consistency manipulation and expectation reached significance, $F$ $(1,67)=8.63, p<.005, \eta^{2}=.11$. Specific comparisons showed, as predicted, that the information given was more surprising $(M=4.59, S D=1.40)$ than expected $(M=3.26, S D=1.42)$, when it referred to culinary school students, $t(33)=2.98, p=.003, d=0.94$. When it referred to business school students it was more expected $(M=4.11, S D=1.65)$ than surprising $(M=3.49, S D=1.73)$, although the difference was not statistically significant, $t(36)=1.25, p=.099$, $d=0.37$. This indicates that the manipulation was effective, because in the inconsistency condition participants perceived the information provided as being inconsistent with their previous knowledge, while in the consistency condition they perceived it as being consistent with that knowledge (or at least as not being inconsistent).

Need for closure and the application of compensatory rules. First, we calculated the indices of change in the perception of both groups on warmth, competence, and morality. We subtracted the first evaluation (before the manipulation) of a group on a given dimension from the second evaluation (after the manipulation). Thus, positive values of the indices indicate that a given group was perceived as being higher on a given dimension in the second evaluation, as compared to the first one. This gives six indexes of change: (3) change in the perception on (warmth vs. competence vs. morality) $\times(2)$ group (business school students vs. culinary school students). To test our hypotheses, we performed regression analyses with the change index of the competence dimension of students from business school as a dependent variable, the change index of the warmth dimension of the assessment of students from the culinary school as a predictor, and NFC and the experimental conditions as moderators. As in the previously described studies, we tested the full model with all main effects, two-way interaction combinations, and a three-way interaction between predictor and two moderators. Moreover, we controlled for the remaining four change indices: the change index in the evaluation of culinary school students on morality, the change index in the evaluation of business school students on morality, the change index in the evaluation of culinary school students on competence, and the change index in the evaluation of business school students on warmth. Before 
computing the interaction terms, the variable identifying the conditions was dummy coded, and all continuous variables were standardized. A positive relationship between the predictor and the DV indicates the application of the compensatory rules (the more culinary school students were perceived as warmer in the posttest, relative to the pretest, the more business school students should be perceived as more competent in posttest, relative to the pretest).

Firstly, we compared the condition of inconsistency and the control condition (coded as 0 control/ 1 inconsistency condition). We expected a significant threeway interaction, as the pattern for individuals high in NFC should differ between these conditions. The model was significant, $F(13,49)=3.00, p=.003$, $R^{2}=.44$. The change score on the warmth dimension for business school students $(\beta=.30 ; t=2.13$, $p=.038,95 \%$ CI $[0.02,0.59])$ was statistically significant. The three-way interaction between NFC, condition, and the predictor (change score on the dimension of warmth for culinary school students) did not reach the level of significance $(\beta=-.93 ; t=1.89, p=.065$, $R^{2}=.04,95 \%$ CI $\left.[-1.93,0.06]\right)$. Although the interaction was not statistically significant at level of $p<.05$, we decided to check whether the predicted pattern occurred. The interaction is depicted in Figure 3.

To probe for this interaction, we used a simple slope analysis and calculated the effect of our predictor on our DV at low $(-1 S D)$ and high $(+1 S D)$ values of the first moderator (NFC), at values of a second moderator (dummy-coded experimental condition). Simple slope analysis showed that in the control condition, a positive relationship between predictor and dependent variable appeared among participants high in NFC $(\beta=.84 ; t=1.83, p=.059,95 \%$ CI $[-0.03,1.72])$, but not among participants low in NFC $(\beta=-.904$; $t=1.58, p=.120,95 \%$ CI $[-2.05,0.25])$. As in Study 2 , in the inconsistent information condition, this pattern changed for participants high in NFC $(\beta=-.23$; $t=.56, p=.578,95 \%$ CI $[-1.07,0.60])$, but for participants low in NFC it remained the same $(\beta=-.11$; $t=.67, p=.506,95 \%$ CI $[-0.45,0.22])$.
We also performed comparisons of the difference between slope coefficients (Cohen, Cohen, West, \& Aiken, 2003). As predicted, the compensatory rules application was stronger among people high in NFC when they did not experience inconsistency, as compared to the situation when they experienced inconsistency $(Z=1.79 ; p=.040)$; and remained the same for people low in NFC $(Z=1.33 ; p=.19)$.

Secondly, we compared the conditions of consistency and control (coded as 0 control/1 consistency condition). We did not expect any differences between these conditions (as in Study 1); rather, we predicted that only the interaction between the change in the perception of the warmth of culinary school students (predictor) and NFC would be statistically significant. The model was non-significant, $F(13,48)=1.86$, $p=.060, R^{2}=.34$. The change score on the competence dimension for culinary school students $(\beta=.36$; $t=2.57, p=.013,95 \%$ CI $[0.08,0.64])$ and a two-way interaction between change score on the dimension of warmth for culinary school students and NFC were statistically significant $(\beta=.84 ; t=2.47, \quad p=.017$, $R^{2}=.08,95 \%$ CI $\left.[0.16,1.52]\right)$. The interaction is presented in Figure 4. Simple slope analysis showed that, again as predicted, irrespective of condition, a positive relationship between predictor and dependent variable appeared among participants high in NFC $(\beta=.90$; $t=3.09, p=.003,95 \%$ CI $[0.31,1.48])$, while among participants low in NFC this relation was negative $(\beta=-.78 ; t=2.69, p=.01,95 \%$ CI $[-1.36,-0.20])$.

In sum, in this study we once again demonstrated that participants high (vs. low) in NFC apply the compensatory rules, here indicated by a positive relationship between change in the perception of one group on one dimension (culinary school students being perceived as warm), and change in the perception of other group on the opposite dimension (business school students being perceived as competent), when the information provided about the group did not violate participants' preexisting, stereotypical knowledge. However, when confronted with expectancy-inconsistent information, participants high in NFC deviated from these rules. As
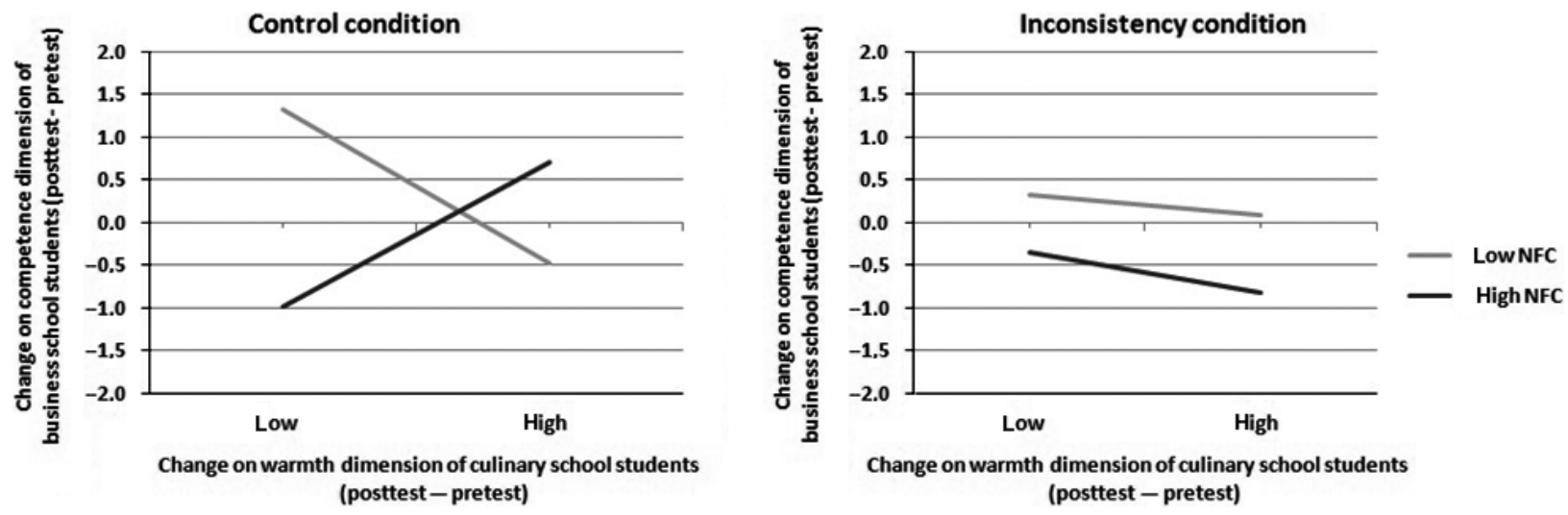

Fig. 3: Regression lines show application of the compensatory rule: change in the perception of business school students on competence dimension (evaluation in pretest subtracted from evaluation in posttest), as a function of change in the perception of culinary school students on warmth dimension (evaluation in pretest subtracted from evaluation in posttest), need for closure (NFC), and experimental condition 


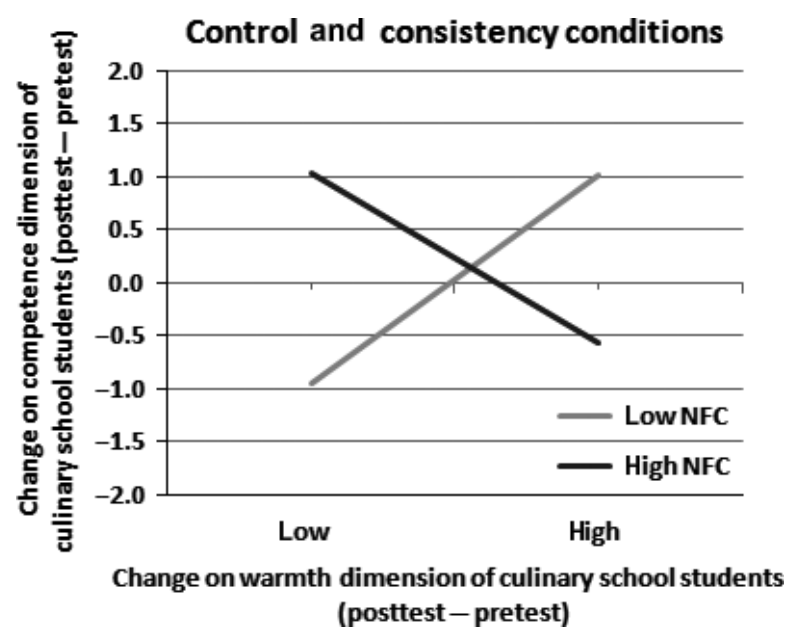

Fig. 4: Regression lines show application of the compensatory rule: change in the perception of business school students on competence dimension (evaluation in pretest subtracted from evaluation in posttest), as a function of change in the perception of culinary school students on warmth dimension (evaluation in pretest subtracted from evaluation in posttest), and need for closure (NFC), in consistency and control conditions

compared to Studies 1 and 2, in this study we captured the more complex and more dynamic nature of the compensatory rules application: How the changing perception of one group affected change in the perception of the other group.

\section{Meta-analysis}

Given that in each study we used the same manipulation and that in all studies we aimed to capture the same phenomenon, that is the application of the compensatory rules, we report the integrated results using a meta-analysis of the three experiments (Cumming, 2014). The meta-analysis was conducted using Comprehensive Meta-Analysis Software, on standardized regression coefficients and its standard errors. The analysis was performed on values of regression coefficients for the predictor, obtained from a simple slope analysis of interaction terms. We analyzed data from three studies, divided into four within study subgroups: 2 (NFC: high vs. low) $\times 2$ (consistency manipulation: consistency vs. inconsistency). We used the random-effects model, as it is appropriate and more realistic in this case (Schmidt, Oh, \& Hayes, 2009). The calculated effect sizes and confidence intervals are reported in Figure 5.

As predicted, the analysis indicated that the compensatory rules were applied only among people high in NFC when there was no inconsistency $(0.85$, $p<.001,95 \% \mathrm{CI}[0.41,1.28])$, while in the remaining groups the effect did not differ significantly from zero, that is for high NFC and inconsistency $(-0.31$, $p=.063,95 \% \mathrm{CI}[-0.63,0.02])$, low NFC and consistency $(-0.18, p=.269,95 \% \mathrm{CI}[-0.49,0.14])$, low NFC and inconsistency $(0.03, p=.832,95 \% \mathrm{CI}[-0.28$, $0.35])$. The results of this meta-analysis support our

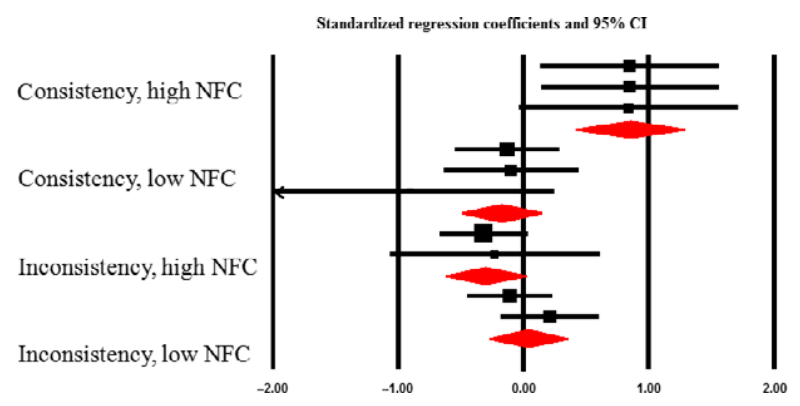

Fig. 5: Meta-analysis of three current studies. Error bars represent 95\% confidence intervals [Colour figure can be viewed at wileyonlinelibrary.com]

prediction that when facing inconsistency, people high in NFC do not apply heuristic tools to establish a relationship between social objects, but they do apply them (here in the form of compensatory rules) when there is no inconsistency involved.

\section{General Discussion}

When operating in familiar situations, people motivated to achieve closure process information based on existing perceptual schemas that efficiently guide interpretation and responses (for an overview, see Roets et al., 2015). Previous studies on the motivation toward closure have focused mainly on stereotypes, overgeneralized beliefs about members of a group that provide people high in NFC with "quick judgments about members of a stereotyped category", thus reducing uncertainty (Kruglanski et al., 2009). In our study, we demonstrated that the compensatory rules may also be a powerful cognitive tool that organizes and simplifies social perception, thus allowing one to reach epistemic goals. When two groups are evaluated, the application of the simple compensatory rules "if warm, then incompetent" and "if cold, then competent" results in perceiving one group as being higher on one dimension, and the other group as being higher on the other dimension. The application of these compensatory rules is a cognitive tool that allows one to achieve a quick and easy solution to the complex task of evaluating social objects on two fundamental dimensions of social perception. Thus, it should be especially useful for people who search for order and predictability. In three studies, we demonstrated that people high in NFC applied these compensatory rules to evaluate two different groups to a greater extent than did people who did not have this motivation (i.e., those low in NFC).

There are studies showing that stereotypes, attitudes, and judgments will likely persist as long as the individual continues to perceive targets in a relatively stable frame of reference (Newcomb, 1950). However, when the unexpected occurs, or when exposed to unfamiliar contexts such as counter-stereotypical information, automatic processing becomes insufficient and people rely on it to a lesser extent (e.g., 
Louis \& Sutton, 1991). In such circumstances, in order to make sense of their new experiences, people may deviate from their default cognitive patterns and begin to notice things they would normally have filtered out (Kossowska, Dragon, \& Bukowski, 2015; Louis \& Sutton, 1991; Tadmor, Hong, Chao, Wiruchnipawan, \& Wang, 2012). In this article, we suggest that expectancy-inconsistent information provided to participants may lead those high in NFC to deviate from the application of compensatory rules, and that this in turn influences their social perception. In all three studies we demonstrated that, when provided with expectancy-consistent information (or in the control condition where no expectation-violating information was provided), people high in NFC applied the compensatory rules to evaluate two different groups to a greater extent than did people low in NFC. However, when individuals high in NFC were provided with information that was expectancy-inconsistent, these effects disappeared. These results are in line with research showing that inconsistency between preexisting knowledge structures and information from the environment (e.g., about one's competence, morality, power position, or the uncontrollability of expected outcomes) leads people to employ stereotypical information less as a means to form impressions about target groups (e.g., Kossowska et al., 2015; Kossowska, Bukowski, et al., 2016; Kossowska, Guinote, \& Strojny, 2016; Rios, Markman, Schroeder, \& Dyczewski, 2014; Tadmor et al., 2012).

Consistency theories and social cognition research argue that expectancy-inconsistent information disrupts the fluency of information processing (Rubin, Paolini, \& Crisp, 2013), can be experienced as threatening, and evokes uncertainty about one's own judgments (Kossowska et al., 2015). We suggest that the feeling of inconsistency may serve as a signal that existing scripts, rules, and other knowledge structures no longer provide guidance on how to act and that they therefore no longer afford a sense of predictability (Ruble, 1994). Further, we claim that inconsistency between previous knowledge and information provided undermines individuals' confidence in their judgmental competence; thus, lessening their reliance on habitual modes of judgment based on compensatory rules. As we have seen, inconsistency affected people high in NFC but not those who were low in NFC. We assume that this is because inconsistency increases the motivation to resolve this inconsistency only among those individuals for whom uncertainty is incompatible with their focal goal, and who are therefore willing to invest effort in changing their default way of information processing, namely people who are high in NFC. In other words, the greater the goal importance (achieving certainty), the greater the likelihood that people will select the most instrumental means, even if they pose considerable demands. As a consequence, people high in NFC who are confronted with expectancy-inconsistent information withdraw from their usual means of achieving closure (i.e., relying on pre-existing knowledge) and attend to, and examine more, information about groups, and ultimately develop more balanced perceptions of groups. Of course, more research is necessary to establish whether our explanation of this process is valid, and what exactly occurs when people experience inconsistency. Our explanation of this mechanism is only one out of many possible alternatives. For example, it is possible that the experience of inconsistency affects a task's perceived characteristics (the task is perceived as being more demanding), and as a consequence, more effort has to be invested in the task. There is also a question regarding the adaptive value of the change in the behavior of people high in NFC. Is this change a strategic response that is made in order to resolve inconsistency, or does the experience of inconsistency simply disorganize the person's regular way of processing information? Future studies should focus more on testing the mechanism responsible for our findings.

Our study also advances NFC theory. The bulk of NFC research has demonstrated that NFC is linked to a preference for a heuristic processing style (for an overview, see Roets et al., 2015). In our opinion, this is unfortunate because it confuses the goal with the means of achieving it. People high in NFC are motivated to achieve the goal of certainty. It just happens that knowledge structures and information processing strategies that are constant, universal, and easy to generalize across different situations are also heuristical, simplistic, and effortless. However, this does not necessarily mean that people high in NFC use these means because they are simplistic. In our opinion they use these means because they are effective tools that allow them to achieve their goal of certainty across many different situations. However, if the situation is perceived to be non-typical, or when the task at hand demands it, people high in NFC are willing to change their preferred means of achieving certainty, which leads to systematic processing (Jaśko, CzernatowiczKukuczka, Kossowska, \& Czarna, 2015; Klein \& Webster, 2000; Kossowska et al., 2015; Kruglanski et al., 1991; Kruglanski, Webster, \& Klem, 1993; Van Hiel \& Mervielde, 2002). In other words, people who are high in NFC would sometimes choose the quickest and proven way to attain closure, which is usually also heuristical and simplistic. However, if this route is made unavailable or unreliable, they are willing to use more systematic processing to attain closure. We would like to challenge the current distinction in existing literature on how high versus low NFC individuals process information, wherein it is in fact possible that high NFC individuals need not always take on the role of a cognitive miser to make decisions.

\section{Conflict of Interest}

The authors confirm they have no conflict of interest to declare. Authors also confirm that this article adheres to ethical guidelines specified in the APA Code 
of Conduct as well as the authors' national ethics guidelines.

\section{Funding}

The preparation of this paper was supported by a grant from the National Science Center (Poland) DEC 2011/ 02/A/HS6/00155 awarded to Małgorzata Kossowska.

\section{Supporting Information}

Additional Supporting Information may be found online in the Supporting Information section at the end of the article.

\section{References}

Abele, A. E., Uchronski, M., Suitner, C., \& Wojciszke, B. (2008). Towards an operationalization of the fundamental dimensions of agency and communion: Trait content ratings in five countries considering valence and frequency of word occurrence. European Journal of Social Psychology, 38, 1202-1217. https://doi.org/10.1002/ejsp. 575

Abele, A. E., \& Wojciszke, B. (2007). Agency and communion from the perspective of self versus others. Journal of Personality and Social Psychology, 93, 751-763. https://doi. org/10.1037/0022-3514.93.5.751

Centrum Badania Opinii Społecznej (CBOS). (2013). Stosunek Polaków do innych narodów [Poles' attitudes towards other nations.] (Research Report). Retrieved from http:// www.cbos.pl/SPISKOM.POL/2013/K_012_13.PDF

Cohen, J., Cohen, P., West, S. G., \& Aiken, L. S. (2003). Applied multiple regression/correlation analysis for the behavioral sciences. New York, NY: Wiley.

Cohn, L. D., \& Becker, B. J. (2003). How meta-analysis increases statistical power. Psychological Methods, 8, 243253. https://doi.org/10.1037/1082-989x.8.3.243

Cumming, G. (2014). The new statistics: Why and how. Psychological Science, 25, 7-29. https://doi.org/10.1177/ 0956797613504966

Dolińska, X., \& Fałkowski, M. (2011). Polska - Niemcy Wzajemny wizerunek $w$ okresie rozszerzania Unii Europejskiej [Poland - Germany. Intergroup perception during extension of European Union] (Research Report). Retrieved from Institute of Public Affairs website http://www.isp. org.pl/files/15646456670594731001117707568.pdf

Driscoll, D. M., Hamilton, D. L., \& Sorrentino, R. M. (1991). Uncertainty orientation and recall of persondescriptive information. Personality and Social Psychology Bulletin, 17, 494-500. https://doi.org/10.1177/0146167 291175003

Fiske, S. T., Cuddy, A. J. C., Glick, P., \& Xu, J. (2002). A model of (often mixed) stereotype content: Competence and warmth respectively follow from perceived status and competition. Journal of Personality and Social Psychology, 82, 878-902. https://doi.org/10.1037//0022-3514. 82.6 .878
Higgins, J. P. T., \& Green, S. (2011). Cochrane handbook for systematic reviews of interventions. Version 5.1.0 [updated March 2011]. The Cochrane Collaboration. Available from www.cochrane-handbook.org

Hoenig, J. M., \& Heisey, D. M. (2001). The abuse of power: The pervasive fallacy of power calculations for data analysis. The American Statistician, 55, 19-24. https://doi.org/ 10.1198/000313001300339897

Houghton, D., \& Grewal, R. (2000). Let's get an answerany answer: Need for consumer cognitive closure. Psychology and Marketing, 17, 911-934. https://doi.org/10. 1002/1520-6793(200011)17

Jaśko, K., Czernatowicz-Kukuczka, A., Kossowska, M., \& Czarna, A. Z. (2015). Individual differences in response to uncertainty and decision making: The role of behavioral inhibition system and need for closure. Motivation and Emotion, 39, 541-552. https://doi.org/10.1007/ s11031-015-9478-x

Kervyn, N., Judd, C. M., \& Yzerbyt, V. Y. (2009). You want to appear competent? Be mean! You want to appear sociable? Be lazy! Group differentiation and the compensation effect. Journal of Experimental Social Psychology, 45, 363-367. https://doi.org/10.1016/j.jesp.2008.08.006

Kervyn, N., Yzerbyt, V. Y., Demoulin, S., \& Judd, C. M. (2008). Competence and warmth in context: The compensatory nature of stereotypic views of national groups. European Journal of Social Psychology, 38, 1175-1183. https://doi.org/10.1002/ejsp

Klein, T. F., \& Webster, D. M. (2000). Individual differences in argument scrutiny as motivated by need for cognitive closure. Basic and Applied Social Psychology, 22, 119-129. https://doi.org/10.1207/s15324834basp2202

Kossowska, M., Bukowski, M., Guinote, A., Dragon, P., \& Kruglanski, A. (2016). Self- image threat decreases stereotyping: The role of motivation toward closure. Motivation $\theta$ Emotion, 40, 830-841. https://doi.org/10. 1007/s11031-016-9582-6

Kossowska, M., Dragon, P., \& Bukowski, M. (2015). When need for closure leads to positive attitudes towards a negatively stereotyped outgroup. Motivation and Emotion, 39, 88-98. https://doi.org/10.1007/ s11031-014-9414-5

Kossowska, M., Guinote, A., \& Strojny, P. (2016). Power boosts reliance on preferred processing styles. Motivation $\theta$ Emotion, 40(4), 556-565. https://doi.org/10.1007/ s11031-016-9548-8

Kruglanski, A. W. (1989). Lay epistemics and human knowledge: Cognitive and motivational bases. New York, NY: Plenum.

Kruglanski, A. W., Dechesne, M., Orehek, E., \& Pierro, A. (2009). Three decades of lay epistemics: The why, how, and who of knowledge formation. European Review of Social Psychology, 20, 146-191. https://doi.org/10.1080/ 10463280902860037

Kruglanski, A. W., Peri, N., \& Zakai, D. (1991). Interactive effects of need for closure and initial confidence on social information seeking. Social Cognition, 9, 127-148. https://doi.org/10.1521/soco.1991.9.2.127

Kruglanski, A. W., Pierro, A., Mannetti, L., Erb, H., \& Chun, W. (2007). On the parameters of human judgment. Advances in Experimental Social Psychology, 39, 255303. https://doi.org/10.1016/s0065-2601(06)39005-3 
Kruglanski, A. W., \& Shteynberg, G. (2012). Cognitive consistency as means to an end: How subjective logic affords knowledge. In B. Gawronski \& F. Strack (Eds.), Cognitive consistency: A fundamental principle in social cognition (pp. 245-264). New York, NY: Guilford.

Kruglanski, A. W., Webster, D. M., \& Klem, A. (1993). Motivated resistance and openness to persuasion in the presence or absence of prior information. Journal of Personality and Social Psychology, 65, 861-876. https://doi.org/ 10.1037/0022-3514.65.5.861

Louis, M. R., \& Sutton, R. I. (1991). Switching cognitive gears: From habits of mind to active thinking. Human Relations, 44, 55-76. https://doi.org/10.1177/001872679 104400104

Luhtanen, R., \& Crocker, J. (1992). A collective self-esteem scale: Self-evaluation of one's social identity. Personality and Social Psychology Bulletin, 18, 302-318. https://doi. org/10.1177/0146167292183006

Mussweiler, T., \& Epstude, K. (2009). Relatively fast! Efficiency advantages of comparative thinking. Journal of Experimental Psychology: General, 138, 1-21. https://doi. org/10.1037/a0014374

Mussweiler, T., \& Posten, A. C. (2011). Relatively certain! Comparative thinking reduces uncertainty. Cognition, 122, 236-240. https://doi.org/10.1016/j.cognition.2011. 10.005

Mussweiler, T., \& Rüter, K. (2003). What friends are for! The use of routine standards in social comparison. Journal of Personality and Social Psychology, 85, 467-481. https://doi.org/10.1037/0022-3514.85.3.467

Neal, D. T., Wood, W., \& Quinn, J. M. (2006). Habits: A repeat performance. Current Directions in Psychological Science, 14(4), 198-202. https://doi.org/10.1111/j.14678721.2006.00435.x

Neuberg, S. L., \& Newsom, J. T. (1993). Personal need for structure: Individual differences in the desire for simple structure. Journal of Personality and Social Psychology, 65, 113-131. https://doi.org/10.1037/0022- 3514.65.1.113

Newcomb, T. M. (1950). Social psychology. New York, NY: Dryden Press.

Rios, K., Markman, K. D., Schroeder, J. R., \& Dyczewski, E. (2014). A (creative) portrait of the uncertain individual: Self-uncertainty and individualism enhance creative generation. Personality and Social Psychology Bulletin, 4, 1-13. https://doi.org/10.1177/0146167214535640

Roets, A., Kruglanski, A. W., Kossowska, M., Pierro, A., \& Hong, Y.-y. (2015). The motivated gatekeeper of our minds: New directions in need for closure theory and research. Advances in Experimental Social Psychology, 52, 221-283. https://doi.org/10.1016/bs.aesp.2015.01.001
Roets, A., \& Van Hiel, A. (2007). Separating ability from need: Clarifying the dimensional structure of the Need for Closure Scale. Personality and Social Psychology Bulletin, 33, 266-280. https://doi.org/10.1177/0146167206294744

Rubin, M., Paolini, S., \& Crisp, R. J. (2013). Linguistic description moderates the evaluations of counterstereotypical people. Social Psychology, 44, 289-298. https://doi. org/10.1027/1864-9335/a000114

Ruble, D. N. (1994). A phase model of transitions: Cognitive and motivational consequences. Advances in Experimental Social Psychology, 26, 163-214. https://doi.org/10. 1016/s00652601(08)60154-9

Rüter, K., \& Mussweiler, T. (2005). Bonds of friendship: Comparative self- evaluations evoke the use of routine standards. Social Cognition, 23, 137-160. https://doi.org/ 10.1521/soco.23.2.137.65624

Schmidt, F. L., Oh, I.-S, \& Hayes, T. L. (2009). Fixed- versus random-effects models in meta-analysis: Model properties and an empirical comparison of differences in results. British Journal of Mathematical $\theta$ Statistical Psychology, 62, 97-128. https://doi.org/10.1348/000711007x255327

Swann, W. B., Gómez, A., Seyle, D. C., Morales, J. F., \& Huici, C. (2009). Identity fusion: The interplay of personal and social identities in extreme group behavior. Journal of Personality and Social Psychology, 96, 995-1011. https://doi.org/10.1037/a0013668

Tadmor, C. T., Hong, Y., Chao, M. M., Wiruchnipawan, F., \& Wang, W. (2012). Multicultural experiences reduce intergroup bias through epistemic unfreezing. Journal of Personality and Social Psychology, 103, 750-772. https://doi. org/10.1037/a0029719

Van Hiel, A., \& Mervielde, I. (2002). The need for closure and the spontaneous use of complex and simple cognitive structures. Journal of Social Psychology, 143, 559-568. https://doi.org/10.1521/soco.20.5.380.21124

Webster, D. M., \& Kruglanski, A. W. (1994). Individual differences in need for cognitive closure. Journal of Personality and Social Psychology, 67, 1049-1062. https://doi.org/ 10.1037/0022-3514.67.6.1049

Wojciszke, B., \& Abele, A. E. (2008). The primacy of communion over agency and its reversals in evaluations. European Journal of Social Psychology, 38, 1139-1147. https://doi.org/10.1002/ejsp.549

Zumbo, B. D., \& Hubley, A. M. (1998). A note on misconceptions concerning prospective and retrospective power. Journal of the Royal Statistical Society, Series D: The Statistician, 47, 385-388. https://doi.org/10.1111/14679884.00139 